\title{
ESTIMATES ON THE MAPPING FUNCTION AND ITS DERIVATIVES IN CONFORMAL MAPPING OF NEARLY CIRCULAR REGIONS $\left({ }^{1}\right)$
}

\author{
BY \\ E. J. SPECHT
}

1. Introduction. Let $C$ be a closed Jordan curve which contains the origin in its interior and which lies in the ring

$$
1 \leqq|w| \leqq 1+\epsilon
$$

where $\epsilon$ is some positive number. Let $w=f(z)$ map the circle $|z|<1$ conformally onto the interior $R$ of $C$ in such a manner that $f(0)=0$ and $f^{\prime}(0)$ $>0$. If $\epsilon$ is "small," then it is to be expected that $w=f(z)$ is "close" to the identity transformation, namely, $w=z$, and the problem of establishing bounds in terms of $\epsilon$ for $|f(z)-z|$ valid in the closed circle $|z| \leqq 1$ has been repeatedly treated in the literature on conformal mapping. The first result in this direction was given by L. Bieberbach $\left({ }^{2}\right)$ in 1924 , and his theorem was sharpened considerably by A. R. Marchenko( $\left.{ }^{3}\right)$ in a short note published in 1935.

In this note Marchenko gives a theorem (without proof) which states that under hypothesis (a) and an additional hypothesis on the smoothness of $C$,

$$
|f(z)-z| \leqq K \epsilon \quad(|z| \leqq 1),
$$

where $K$ is constant. As a first result in the present paper a simple proof of this theorem is given which not only shows the existence of the constant $K$ but also give a numerical estimate for $K$. (See Theorem I, §2.)

In view of the fact that "nearly circular" regions play an important part in the theory as well as in some of the applications of conformal mapping, parcularly in connection with the numerical computation of the mapping function of aerodynamics $\left({ }^{4}\right)$, it seemed of some interest to pursue this problem

Presented to the Society, September 2, 1949; received by the editors August 26, 1950 and, in revised form, December 22, 1950.

(1) The material of this paper forms part of a Ph.D. thesis prepared under Professor S. E. Warschawski of the University of Minnesota.

(2) L. Bierbach [1]. Numbers in brackets refer to the bibliography at the end of the paper.

(3) A. R. Marchenko [4]. Marchenko's result gives the best possible bound as far as the power of $\epsilon$ is concerned. Compare also J. Ferrand [2] where a new proof of Marchenko's theorem is announced.

(4) See, for example, Höhndorf [3], Theodorsen [8], Theodorsen and Garrick [9], and Warschawski [12]. Höhndorf applies Bieberbach's theorem mentioned above in the solution of an engineering problem for actual numerical estimates. 
further and to investigate the analogous questions for the derivatives of the mapping function. The problem considered is that of determining actual numerical bounds for $\left|f^{\prime}(z)-1\right|,\left|f^{\prime \prime}(z)\right|, \cdots,\left|f^{(n)}(z)\right|$ assuming, of course, further suitable restrictions on the curve $C$.

\section{Statements of results.}

TheOREM I (MARChenko). Let $C$ be a closed Jordan curve which is starshaped $\left.{ }^{5}\right)$ with respect to the origin and let the polar equation of $C$ be $\rho=\rho(\phi)$, $0 \leqq \phi \leqq 2 \pi$, where $\rho(\phi)$ is periodic of period $2 \pi$ and satisfies the following additional hypotheses:

(a) For some $\epsilon>0$

$$
1 \leqq \rho(\phi) \leqq 1+\epsilon, \quad 0 \leqq \phi \leqq 2 \pi .
$$

(b) There exists a positive number( $\left.{ }^{6}\right) M$ such that

$$
|\rho(\phi+\delta)-\rho(\phi)| \leqq M \in|\delta|,
$$

where $0 \leqq \phi \leqq 2 \pi$ and $\delta$ is any real number. Let $w=f(z)$ map $|z|<1$ conformally onto the interior of $C$ in such a manner that $f(0)=0$ and $f^{\prime}(0)>0$. Then there exists a constant $K$ which depends only on $M$ such that for $|z| \leqq 1$

$$
|f(z)-z| \leqq K, \epsilon .
$$

In fact $K \leqq 1+M(2 \log 2+1)$ if $\epsilon \leqq 1$.

THEOREM II. Let $C$ be a closed Jordan curve which is star-shaped with respect to the origin. Let the polar equation of $C$ be $\rho=\rho(\phi)$, where $\rho(\phi)$ is positive, periodic of period $2 \pi$, and satisfies the following additional hypotheses:

(a) $\rho^{\prime}(\phi)$ is continuous and

$$
\left|\rho^{\prime}(\phi) / \rho(\phi)\right|<\epsilon \quad(0<\epsilon<1) .
$$

(b) For all $\phi_{0}, 0 \leqq \phi_{0} \leqq 2 \pi$,

$$
\frac{1}{2 \pi} \int_{-\pi}^{\pi}\left|\frac{\rho^{\prime}(\phi) / \rho(\phi)-\rho^{\prime}\left(\phi_{0}\right) / \rho\left(\phi_{0}\right)}{\sin \left(\left(\phi-\phi_{0}\right) / 2\right)}\right| d \phi<\delta \quad(0<\delta<1)
$$

the integral being uniformly convergent $\left(^{7}\right)$ in this interval. Let $f(z)$ be defined as in Theorem I and let $\phi(\theta)=\arg f\left(e^{i \theta}\right)$. Then $f^{\prime}(z)$ exists and is continuous for $|z| \leqq 1$. Furthermore,

$$
\left|\phi^{\prime}(\theta)-1\right| \leqq \frac{\delta+\epsilon^{2}}{1-\delta}
$$

(5) A closed curve is star-shaped with respect to the origin if every ray from the origin intersects the curve in exactly one point.

( $\left.{ }^{*}\right)$ That is, $\rho(\phi)$ satisfies a Lipschitz condition with Lipschitz constant $M \epsilon$.

( ${ }^{7}$ That is, given $\eta>0$, there exists a number $a>0$, depending only on $\eta$ and not on $\phi_{0}$, such that $\phi_{0}-x<a, y-\phi_{0}<a$, and $x<y$ implies $\int_{x}^{y}<\eta$. 
and

$$
\left|z \frac{f^{\prime}(z)}{f(z)}-1\right| \leqq \frac{\epsilon+\epsilon^{2}+\epsilon^{3}+\delta}{1-\delta}=k(\epsilon) \quad(|z| \leqq 1) .
$$

THEOREM III. If in addition to all the hypotheses of Theorem II, the curve $C$ is assumed to lie in the ring

$$
1 \leqq|w|<1+\epsilon
$$

where $\epsilon$ is the number given in hypothesis (a) of Theorem II, and if $f(z)$ is defined as in Theorem I, then

$$
\left|f^{\prime}(z)-1\right| \leqq 5 \frac{\epsilon+\epsilon^{2}+\epsilon^{3}+\delta}{1-\delta} \quad(|z| \leqq 1) .
$$

THEOREM IV. Let $C$ be a closed Jordan curve which is star-shaped with respect to the origin and let the polar equation of $C$ be $\rho=\rho(\phi)$ where $\rho(\phi)$ is positive, periodic of period $2 \pi$, and satisfies the following additional hypotheses:

(a) $p(\phi)=(d / d \phi)\left(\rho^{\prime}(\phi) / \rho(\phi)\right)$ exists and is continuous and

$$
|p(\phi)|<\epsilon / \pi \quad(0<\epsilon<1) .
$$

(b) For all $\phi_{0}, 0 \leqq \phi_{0} \leqq 2 \pi$,

$$
\frac{1}{2 \pi} \int_{-\pi}^{\pi}\left|\frac{\omega^{\prime}(\phi)-\omega^{\prime}\left(\phi_{0}\right)}{\sin \left(\left(\phi-\phi_{0}\right) / 2\right)}\right| d \phi \leqq \epsilon
$$

where $\omega(\phi)=-\arctan \left(\rho^{\prime}(\phi) / \rho(\phi)\right)$ (the principal branch of the arc tangent being chosen). Let $f(z)$ and $\phi(\theta)$ be defined as in Theorem II, then $f^{\prime \prime}(z)$ exists for $|z| \leqq 1$,

$$
\left|\frac{\phi^{\prime \prime}(\theta)}{\phi^{\prime}(\theta)}\right| \leqq \epsilon\left(\frac{1+\epsilon^{2}}{1-\left(8 / \pi^{2}\right) \epsilon}\right)\left(1+\frac{2}{\pi} \epsilon\right)
$$

and

$$
\begin{aligned}
\left|\frac{f^{\prime \prime}(z)}{f^{\prime}(z)}\right| & \leqq \epsilon \frac{(3+(1 / \pi))+\epsilon\left(\left(\left(1+\epsilon+\epsilon^{2}\right) / \pi\right)+1+2 \epsilon\right)}{1-\left(8 / \pi^{2}\right) \epsilon} \\
& <\epsilon \frac{3+(1 / \pi)+4 \epsilon}{1-\left(8 / \pi^{2}\right) \epsilon} .
\end{aligned}
$$

THEOREM V. Let $C$ be a closed Jordan curve which is star-shaped with respect to the origin and let the polar equation of $C$ be $\rho=\rho(\phi), 0 \leqq \phi \leqq 2 \pi$, where $\rho(\phi)$ is positive, periodic of period $2 \pi$, and satisfies the following additional hypotheses:

(a) $\rho^{(n)}(\phi)$ exists and is continuous, 


$$
|p(\phi)|<\epsilon / \pi
$$

where $p(\phi)=(d / d \phi)\left(\rho^{\prime}(\phi) / \rho(\phi)\right)$, and

$$
\left|\omega^{(k)}(\phi)\right|<\epsilon / \pi \quad \text { for } k=2, \cdots, n-1,
$$

where $\omega(\phi)=-\operatorname{arc} \tan \left(\rho^{\prime}(\phi) / \rho(\phi)\right)$ (the principal value of the arc tangent being chosen).

(b) For all $\phi_{0}, 0 \leqq \phi_{0} \leqq 2 \pi$,

$$
\frac{1}{2 \pi} \int_{-\pi}^{\pi}\left|\frac{\omega^{(n-1)}(\phi)-\omega^{(n-1)}(\phi)_{0}}{\sin \left(\left(\phi-\phi_{0}\right) / 2\right)}\right| d \phi \leqq \epsilon .
$$

Let $f(z)$ and $\phi(\theta)$ be defined as in Theorem II, then $f^{(n)}(z)$ exists for $|z| \leqq 1$ and there exist two absolute constants $K_{n}$ and $J_{n}^{\prime}$, that is, numbers which depend only on $n$, but not on $\epsilon$ or on the curve $\left({ }^{8}\right) C$, such that

$$
\left|\phi^{(n)}(\theta)\right| \leqq K_{n} \epsilon
$$

and

$$
\left|f^{(n)}(z) / f^{\prime}(z)\right| \leqq J_{n}^{\prime} \quad(|z| \leqq 1, n \geqq 2) .
$$

REMARK. If in addition to the hypotheses of Theorem $\mathrm{V}$ we assume that $C$ lies in the ring

$$
1 \leqq|w|<1+\epsilon \quad(0<\epsilon<1),
$$

then by Theorem III (since $\delta$ may be taken as $\left(8 / \pi^{2}\right) \epsilon$, see Preliminary inequalities under \$5) there exists an absolute constant $J_{1}$ such that

$$
\left|f^{\prime}(z)-1\right| \leqq J_{1 \epsilon} \text { or }\left|f^{\prime}(z)\right| \leqq J_{1} \epsilon+1<J_{1}+1 .
$$

Hence under this additional assumption

$$
\left|f^{(n)}(z)\right| \leqq\left(J_{1}+1\right) J_{n}^{\prime} \epsilon=J_{n} \epsilon
$$

where $J_{n}=\left(J_{1}+1\right) J_{n}^{\prime}(n=2,3, \cdots)$.

3. Proof of Theorem I.

(A) An integral representation for $\arg (f(z) / z)$ on the boundary. Let $C$ be a closed star-shaped Jordan curve, containing the origin, whose polar equation is $\rho=\rho(\phi), 0 \leqq \phi \leqq 2 \pi$, where $\rho(\phi)$ is positive and has bounded difference quotients. Let the function $w=f(z)$ map $|z|<1$ conformally onto the interior of $C$ in such a manner that $f(0)=0$ and $f^{\prime}(0)>0$. Let $\phi(\theta)-\theta=\arg (f(z) / z)$, $z=e^{i \theta}$, where the branch of $\arg (f(z) / z)$ is chosen so that $[\arg (f(z) / z)]_{z=0}$ $=\arg f^{\prime}(0)=0$, and let $t(\phi)$ denote the inverse function of $\phi(\theta)$. Then for all

(8) Thus the inequalities (2.8) and (2.9) are satisfied with the same $K_{n}$ and $J_{n}^{\prime}$ for the mapping function of every curve $C$ for which hypotheses (a) and (b) are fulfilled. 
$\theta$ for which $\phi(\theta)$ and $\rho(\phi(\theta))$ have $\left(^{9}\right)$ a (finite) derivative and $\phi^{\prime}(\theta) \neq 0$, that is, for almost all $\theta$,

$$
\phi(\theta)-\theta=\frac{1}{\pi} \int_{-\pi}^{\pi} \log \left|\sin \frac{t(\phi)-\theta}{2}\right| \frac{\rho^{\prime}}{\rho}[\phi] d \phi .
$$

Remark. Since $t(\phi)-\theta \sim\left(\phi-\phi_{0}\right) \cdot t^{\prime}\left(\phi_{0}\right)$ as $\phi \rightarrow \phi_{0}$, where $\phi_{0}=\phi(\theta)$, and $\left(\rho^{\prime} / \rho\right)[\phi]$ is bounded, the integral of (3.1) exists in the sense of Lebesgue.

Proof. Let $\theta$ be a value for which $\phi^{\prime}(\theta)$ and $\rho^{\prime}(\phi)(\phi=\phi(\theta))$ exist and $\phi^{\prime}(\theta) \neq 0$. Since $\phi(\theta)-\theta$ is the imaginary part of $\log (f(z) / z), z=e^{i \theta}$, where $\log (f(z) / z)$ is the $\operatorname{real}\left({ }^{10}\right) \log f^{\prime}(0)$ when $z=0$, we have

$$
\phi(\theta)-\theta=-\frac{1}{2 \pi} \int_{\theta-\pi}^{\theta+\pi}[\log \rho(\phi(t))-\log \rho(\phi(\theta))] \cot \frac{t-\theta}{2} d t .
$$

Since $\rho(\phi)$ is continuous and has bounded difference quotients, it follows that $C$ is rectifiable, and therefore, by a theorem of $\mathrm{F}$. and M. Riesz (see $\mathrm{F}$. and M. Riesz [5]), that $\phi(\theta)$ is an absolutely continuous (and monotonic) function. Hence $\rho(\phi(\theta))$ is an absolutely continuous function of $\theta$, for all $\theta$. We shall make use of this fact in changing the last integral by an integration by parts. The function

$$
\Phi(t)=[\log \rho(\phi(t))-\log \rho(\phi(\theta))] \log |\sin (t-\theta) / 2|,
$$

for $t \neq 0, \Phi(0)=0$, is continuous for $\theta-\pi \leqq t \leqq \theta+\pi$. Hence

$$
\begin{aligned}
\phi(\theta)-\theta= & -\frac{1}{\pi}\left[\left(\log \rho(\phi(t)-\log \rho(\phi(\theta))) \log \left|\sin \frac{t-\theta}{2}\right|\right]_{\theta-\pi}^{\theta+\pi}\right. \\
& +\lim _{\delta \rightarrow 0}\left[\int_{\theta+\delta}^{\theta+\pi} \frac{\rho^{\prime}}{\rho}[\phi(t)] \phi^{\prime}(t) \log \left|\sin \frac{t-\theta}{2}\right| d t\right. \\
& \left.+\int_{\theta-\pi}^{\theta-\delta} \frac{\rho^{\prime}}{\rho}[\phi(t)] \phi^{\prime}(t) \log \left|\sin \frac{t-\theta}{2}\right| d t\right],
\end{aligned}
$$

the integrated part being zero because of the periodicity of $\Phi(t)$. Making the substitution $t=t(\phi), \phi^{\prime}(t) d t=d \phi$, and observing the remark concerning the existence of the integral of (3.1), we obtain the formula (3.1).

(B) Estimate of $\left|\arg f\left(e^{i \theta}\right)-\theta\right|$ and $|f(z)-z|$. Let $\phi(\theta)-\theta=\arg (f(z) / z)$, $z=e^{i \theta}$, where $\arg (f(z) / z)$ is so chosen that $\arg f^{\prime}(0)=0$. Let $\theta$ be a value for which $\phi^{\prime}(\theta)$ and $\rho^{\prime}(\phi)$ exist and $\phi^{\prime}(\theta) \neq 0$. Then by (3.1)

$$
\phi(\theta)-\theta=\frac{1}{\pi} \int_{\theta-\pi}^{\theta+\pi} \log \left|\sin \frac{t-\theta}{2}\right| \frac{\rho^{\prime}}{\rho}[t] \phi^{\prime}(t) d t .
$$

(9) The notation $\left(\rho^{\prime} / \rho\right)[\phi]$ means $\rho^{\prime}(\phi) / \rho(\phi)$.

(10) That is, let $F(z)=\log (f(z) / z)$ for $z \neq 0, F(0)=\log f^{\prime}(0)$, and let $F(0)$ be real. 
Since $\phi^{\prime}(\theta)>0$ and $\left|\left(\rho^{\prime} / \rho\right)[\phi]\right| \leqq M \epsilon$ (by hypothesis (b)) we have

$$
\begin{aligned}
|\phi(\theta)-\theta| \leqq M \epsilon & \frac{1}{\pi} \int_{\theta-\pi}^{\theta+\pi}\left(-\log \left|\sin \frac{t-\theta}{2}\right|\right) \phi^{\prime}(t) d t \\
= & M \epsilon\left[\frac{1}{\pi} \int_{\theta-\pi}^{\theta+\pi}\left(-\log \left|\sin \frac{t-\theta}{2}\right|\right)\left(\phi^{\prime}(t)-1\right) d t\right. \\
& \left.-\frac{1}{\pi} \int_{\theta-\pi}^{\theta+\pi} \log \left|\sin \frac{t-\theta}{2}\right| d t\right] .
\end{aligned}
$$

The second integral has the value $-2 \pi \log 2$. The first integral is easily evaluated by integration by parts. We have

$$
\begin{aligned}
-\frac{1}{\pi} \int_{\theta-\pi}^{\theta+\pi} \log \left|\sin \frac{t-\theta}{2}\right|\left(\phi^{\prime}(t)-1\right) d t \\
\quad=\frac{1}{2 \pi} \int_{\theta-\pi}^{\theta+\pi}\left(\cot \frac{t-\theta}{2}\right)(\phi(t)-t-[\phi(\theta)-\theta]) d t=\log \left|\frac{f\left(e^{i \theta}\right)}{f^{\prime}(0)}\right| .
\end{aligned}
$$

It follows( ${ }^{(1)}$ from hypothesis (a) that

Hence we obtain

$$
\left|f(z) / f^{\prime}(0)\right| \leqq 1+\epsilon \quad \text { for }|z| \leqq 1 .
$$

$$
|\phi(\theta)-\theta| \leqq M \epsilon(2 \log 2+\epsilon) .
$$

This holds for almost all $\theta$, and hence by the continuity of $\phi(\theta)-\theta$ it holds for all $\theta, 0 \leqq \theta \leqq 2 \pi$.

Let $\rho=\rho(\phi(\theta))$, then by hypothesis (a) and by (3.2)

$$
\begin{aligned}
\left|\frac{f(z)}{z}-1\right|=\left|\rho e^{i[\phi(\theta)-\theta]}-1\right| & \leqq|\rho-1|+\left|e^{i[\phi(\theta)-\theta]}-1\right| \\
& \leqq \epsilon+M \epsilon(2 \log 2+\epsilon) .
\end{aligned}
$$

Thus, if $\epsilon \leqq 1$ and $|z|=1,|f(z)-z| \leqq K \epsilon$ where $K \leqq 1+M(2 \log 2+1)$. By the maximum-modulus principle this holds for $|z|<1$.

4. Proof of Theorems II and III.

(A) An integral representation for $(d / d \theta) \arg f\left(e^{i \theta}\right)$. Let $C$ be a closed starshaped Jordan curve, containing the origin, whose polar equation is $\rho=\rho(\phi)$, $0 \leqq \phi \leqq 2 \pi$, where $\rho(\phi)$ is positive, $\rho^{\prime}(\phi)$ is continuous, and the integral

$$
\int_{\phi_{0}-\pi}^{\phi_{0}+\pi}\left|\frac{\left(\rho^{\prime} / \rho\right)[\phi]-\left(\rho^{\prime} / \rho\right)\left[\phi_{0}\right]}{\phi-\phi_{0}}\right| d \phi
$$

exists for some $\phi_{0}$. Let $f(z)$ and $\phi(t)=\arg f\left(e^{i t}\right)$ be defined as in Theorem I and

(11) Applying the minimum-modulus principle to the function $g(z)=(f(z) / z), z \neq 0, g(0)$ $=f^{\prime}(0)$, we see that hypothesis (a) implies $f^{\prime}(0) \geqq 1$. 
let $t(\phi)$ be the inverse function of $\phi(t)$. If $\theta=t\left(\phi_{0}\right)$, then $\phi^{\prime}(\theta)$ exists and

$$
\begin{aligned}
\phi(\theta)\left[1+\left(\frac{\rho^{\prime}}{\rho}\left[\phi_{0}\right]\right)^{2}\right] & \\
& =1-\frac{1}{2 \pi} \int_{-\pi}^{\pi}\left(\cot \frac{t(\phi)-\theta}{2}\right)\left(\frac{\rho^{\prime}}{\rho}[\phi]-\frac{\rho^{\prime}}{\rho}\left[\phi_{0}\right]\right) d \phi .
\end{aligned}
$$

Proof. Because of hypothesis (4.1) the function $f(z)$ has a (nonvanishing) derivative $\left({ }^{12}\right) f^{\prime}\left(z_{0}\right)$ at $z_{0}=e^{i \theta}$, and $\lim _{r \rightarrow 1} f^{\prime}\left(r e^{i \theta}\right)=f^{\prime}\left(z_{0}\right)$. Let $\log (f(z) / z)$ denote the branch of the logarithm which is equal to the real $\log f^{\prime}(0)$ when $z=0$. This function is analytic for $|z|<1$ and has the continuous boundary function $\log \rho(\phi(t))+i(\phi(t)-t)$ for $z=e^{i t}$. Furthermore, $(d / d t)(\log \rho(\phi(t))$ $+i(\phi(t)-t))$ exists for $t=\theta$ and

$$
\lim _{r \rightarrow 1}\left[\frac{\partial}{\partial \alpha} \log \left(\frac{f\left(r e^{i \alpha}\right)}{r e^{i \alpha}}\right)\right]_{\alpha=\theta}=\frac{\rho^{\prime}}{\rho}(\phi(\theta)) \phi^{\prime}(\theta)+i\left[\phi^{\prime}(\theta)-1\right] .
$$

The function $\log (f(z) / z)$ may be represented for $|z|=\left|r e^{i \alpha}\right|<1$ by means of the Poisson integral

$$
\log \frac{f(z)}{z}=\frac{1}{2 \pi} \int_{-\pi}^{\pi}(\log \rho(\phi(t))+i[\phi(t)-t]) \frac{1-r^{2}}{1+r^{2}-2 r \cos (t-\alpha)} d t .
$$

Hence

$$
\begin{aligned}
\frac{\partial}{\partial \alpha}\left(\log \frac{f\left(r e^{i \alpha}\right)}{r e^{i \alpha}}\right) & \\
\quad= & \frac{1}{2 \pi} \int_{-\pi}^{\pi}(\log \rho(\phi(t))+i[\phi(t)-t]) \frac{\partial}{\partial \alpha}\left(\frac{1-r^{2}}{1+r^{2}-2 r \cos (t-\alpha)}\right) d t \\
& =-\frac{1}{2 \pi} \int_{-\pi}^{\pi}(\log \rho(\phi(t))+i[\phi(t)-t]) \frac{\partial}{\partial t}\left(\frac{1-r^{2}}{1+r^{2}-2 r \cos (t-\alpha)}\right) d t .
\end{aligned}
$$

Since $\phi(t)-t$ and $\rho(\phi(t))$ are absolutely continuous and periodic functions of $t$, the period being $2 \pi$, we obtain by an integration by parts

$$
\begin{array}{r}
\frac{\partial}{\partial \alpha}\left(\log \frac{f\left(r e^{i \alpha}\right)}{r e^{i \alpha}}\right) \\
=\frac{1}{2 \pi} \int_{-\pi}^{\pi}\left(\frac{\rho^{\prime}}{\rho}[\phi(t)] \phi^{\prime}(t)+i\left[\phi^{\prime}(t)-1\right]\right) \frac{1-r^{2}}{1+r^{2}-2 r \cos (t-\alpha)} d t \\
(0 \leqq r<1) .
\end{array}
$$

Because of this representation and because $(d / d t)[\rho(\phi(t))+i(\phi(t)-t)]_{t=\theta}$

(12) That is, $\left.\lim _{z^{\prime} \rightarrow z_{0}}\left(f(z)-f\left(z_{0}\right)\right) /\left(z-z_{0}\right)\right)=f^{\prime}\left(z_{0}\right)$ exists when $z \rightarrow z_{0}$ in $|z| \leqq 1\left(z \neq z_{0}\right)$. 
exists, the real and imaginary parts of the boundary function (4.3) are given by the following integrals $\left({ }^{13}\right)$ :

$$
\frac{\rho^{\prime}}{\rho}[\phi(\theta)] \phi^{\prime}(\theta)=\frac{1}{2 \pi} \int_{0}^{\pi}\left[\phi^{\prime}(\theta+\tau)-\phi^{\prime}(\theta-\tau)\right] \cot \frac{\tau}{2} d \tau
$$

and

$\phi^{\prime}(\theta)-1=-\frac{1}{\pi} \int_{0}^{\pi}\left(\left[\frac{\rho^{\prime}}{\rho}[\phi(t)] \phi^{\prime}(t)\right]_{t=\theta+\tau}-\left[\frac{\rho^{\prime}}{\rho}[\phi(t)] \phi^{\prime}(t)\right]_{t=\theta-\tau}\right) \cot \frac{\tau}{2} d \tau$

where both integrals converge in the sense that $\lim _{\eta \rightarrow 0} \int_{\eta}^{\pi}$ exists. We may write

$$
\begin{aligned}
\phi^{\prime}(\theta)-1= & -\frac{1}{2 \pi} \int_{0}^{\pi}\left(\frac{\rho^{\prime}}{\rho}[\phi(\theta+\tau)]-\frac{\rho^{\prime}}{\rho}[\phi(\theta)]\right) \phi^{\prime}(\theta+\tau) \cot \frac{\tau}{2} d \tau \\
& +\frac{1}{2 \pi} \int_{0}^{\pi}\left(\frac{\rho^{\prime}}{\rho}[\phi(\theta-\tau)]-\frac{\rho^{\prime}}{\rho}[\phi(\theta)]\right) \phi^{\prime}(\theta-\tau) \cot \frac{\tau}{2} d \tau \\
& -\frac{1}{2 \pi} \frac{\rho^{\prime}}{\rho}[\phi(\theta)] \int_{0}^{\pi}\left[\phi^{\prime}(\theta+\tau)-\phi(\theta-\tau)\right] \cot \frac{\tau}{2} d \tau .
\end{aligned}
$$

For, the first two integrals exist in the sense of Lebesgue because of the hypothesis (4.1) and the fact that $\phi^{\prime}(t)$ exists almost everywhere, as is seen by introducing $x=\phi(\theta+t)$ and $x=\phi(\theta-t)$, respectively, as variables of integration. If we make this substitution and use (4.4) we obtain (4.2).

(B) Proof of Theorem II. Because of the continuity of $\rho(\phi)$ and the hypothesis (b), $\phi^{\prime}(\theta)$ exists and is continuous $\left({ }^{14}\right)$ for $0 \leqq \theta \leqq 2 \pi$. By (4.2), if $\theta=t\left(\phi_{0}\right)$,

$$
\begin{aligned}
\left(\phi^{\prime}(\theta)-1\right)[1 & \left.+\left(\frac{\rho^{\prime}}{\rho}\left[\phi_{0}\right]\right)^{2}\right] \\
& =-\frac{1}{2 \pi} \int_{-\pi}^{\pi}\left(\cot \frac{t(\phi)-\theta}{2}\right)\left(\frac{\rho^{\prime}}{\rho}[\phi]-\frac{\rho^{\prime}}{\rho}\left[\phi_{0}\right]\right) d \phi-\left(\frac{\rho^{\prime}}{\rho}\left[\phi_{0}\right]\right)^{2} .
\end{aligned}
$$

Choose $\phi_{1}$ so that $t\left(\phi_{1}\right)-\theta=\pi$ and $\phi_{1}-2 \pi \leqq \phi_{0}<\phi_{1}$, then $t\left(\phi_{1}-2 \pi\right)-\theta=-\pi$, and $\phi_{1}-2 \pi \leqq \phi \leqq \phi_{1}$ implies $|t(\phi)-\theta| \leqq \pi$, which in turn implies $\left({ }^{15}\right)$

$$
\left|\cot \frac{t(\phi)-\theta}{2}\right| \leqq \frac{2}{|t(\phi)-\theta|} .
$$

(13) This follows from a theorem on conjugate functions, see A. Zygmund, Trigonometric series, p. $54, \$ 3.45$.

(14) This follows from Warschawski [10, Theorem 10, p. 433].

(15) Note that for $0<x \leqq \pi / 2, x \cot x<1$. 
Letting $\mu=\max \left|\phi^{\prime}(\theta)-1\right|$ and taking the limits in the above integral as $\phi_{1}-2 \pi$ and $\phi_{1}$, we have by (4.5) and hypothesis (a)

$$
\begin{aligned}
\left|\phi^{\prime}(\theta)-1\right| & \leqq \frac{1}{2 \pi} \int_{\phi_{1}-2 \pi}^{\phi_{1}}(\mu+1)\left|\frac{\left(\rho^{\prime} / \rho\right)[\phi]-\left(\rho^{\prime} / \rho\right)\left[\phi_{0}\right]}{\left(\phi-\phi_{0}\right) / 2}\right| d \phi+\epsilon^{2} \\
& \leqq \frac{\mu+1}{2 \pi} \int_{\phi_{0}-\pi}^{\phi_{0}+\pi}\left|\frac{\left(\rho^{\prime} / \rho\right)[\phi]-\left(\rho^{\prime} / \rho\right)\left[\phi_{0}\right]}{\sin \left(\left(\phi-\phi_{0}\right) / 2\right)}\right| d \phi+\epsilon^{2}
\end{aligned}
$$

for $0 \leqq \theta \leqq 2 \pi$. Hence by hypothesis (b)

which implies (2.1).

$$
\mu \leqq(\mu+1) \delta+\epsilon^{2}
$$

In order to prove (2.2) observe that, for $z=e^{i \theta}$,

$$
\begin{aligned}
\left|\frac{d}{d \theta} \log \frac{f(z)}{z}\right| & =\left|i\left(\frac{z f^{\prime}(z)}{f(z)}-1\right)\right|=\left|\frac{\rho^{\prime}}{\rho}[\phi(\theta)] \phi^{\prime}(\theta)+i\left(\phi^{\prime}(\theta)-1\right)\right| \\
& \leqq \epsilon\left(1+\frac{\delta+\epsilon^{2}}{1-\delta}\right)+\frac{\delta+\epsilon^{2}}{1-\delta}=\frac{\epsilon+\epsilon^{2}+\epsilon^{3}+\delta}{1-\delta}=k(\epsilon) .
\end{aligned}
$$

Since the function $z f^{\prime}(z) / f(z)-1$ is analytic for $|z|<1$, if defined as 0 for $z=0$, and continuous for $|z| \leqq 1$, this proves (2.2) for all $|z| \leqq 1$.

(C) Proof of Theorem III. We first note that for $|z| \leqq 1$ :

$$
\left|f^{\prime}(z)-1\right| \leqq\left|\frac{f(z)}{z}\right|\left|z \frac{f^{\prime}(z)}{f(z)}-1\right|+\left|\frac{f(z)}{z}-f^{\prime}(0)\right|+\left|f^{\prime}(0)-1\right| .
$$

Since $f(z) / z$ is analytic for $|z|<1$ if defined as $f^{\prime}(0)$ when $z=0,(2.3)$ implies that

$$
1 \leqq\left|\frac{f(z)}{z}\right|<1+\epsilon
$$

for $|z| \leqq 1$. Hence we have from (2.2) (note that $f^{\prime}(0)>0$ )

$$
\left|f^{\prime}(z)-1\right| \leqq k(\epsilon)[1+\epsilon]+\left|\frac{f(z)}{z}-f^{\prime}(0)\right|+\epsilon .
$$

Now

$$
\left|\frac{f(z)}{z}-f^{\prime}(0)\right|=\left|\int_{0}^{z} \frac{d}{d t}\left(\frac{f(t)}{t}\right) d t\right|=\left|\int_{0}^{z} \frac{f(t)}{t^{2}}\left(t \frac{f^{\prime}(t)}{f(t)}-1\right) d t\right| .
$$

Since the function $t f^{\prime}(t) / f(t)-1$ is regular for $|t|<1$ if defined as 0 when $t=0$, (2.2) implies $\left|(1 / t)\left(t f^{\prime}(t) / f(t)-1\right)\right| \leqq k(\epsilon)$ for $|t| \leqq 1$, and thus that $\left|f(z) / z-f^{\prime}(0)\right| \leqq k(\epsilon)[1+\epsilon]$. We obtain, therefore, from (4.6)

$$
\left|f^{\prime}(z)-1\right| \leqq 2 k(\epsilon)[1+\epsilon]+\epsilon<5 k(\epsilon) \text {. }
$$




\section{Proof of Theorem IV.}

(A) A formula for $\phi^{\prime \prime}(\theta)$. We assume the hypotheses of Theorem IV satisfied. The function $F(z)=\log \left(z f^{\prime}(z) / f(z)\right)$ when $z \neq 0, F(0)=0$, is analytic for $|z|<1$ and continuous for $|z| \leqq 1$. Since $\rho^{\prime \prime}(\phi)$ exists and is continuous and $p(\phi)$ satisfies hypothesis (b), $f^{\prime}(z)$ and hence $F(z)$ has a derivative at every point of the unit circle $\left.{ }^{16}\right)$ (that is, $\lim _{z \rightarrow z_{0}}\left[\left(F(z)-F\left(z_{0}\right)\right) /\left(z-z_{0}\right)\right]$ exists, $\left.\left|z_{0}\right|=1,|z| \leqq 1, z \neq z_{0}\right)$. Hence $(d / d \theta)\left(F\left(e^{i \theta}\right)\right)$ and $\phi^{\prime \prime}(\theta)$ exist for $0 \leqq \theta \leqq 2 \pi$, and $\lim _{r \rightarrow 1}(\partial / \partial \theta)\left(F\left(r e^{i \theta}\right)=(d / d \theta)\left(F\left(e^{i \theta}\right)\right)\right.$. Let $\omega=-\arctan \left(\rho^{\prime} / \rho\right)[\phi]$, where the principal value of the arc tangent is chosen. Then, it is easily seen that

$$
\arg \left[\frac{z f^{\prime}(z)}{f(z)}\right]_{z=e^{i \theta}}=-\arctan \frac{\rho^{\prime}}{\rho}[\phi(\theta)]=\omega(\phi(\theta)) .
$$

Hence

$$
\frac{d}{d \theta} \arg \left[\frac{z f^{\prime}(z)}{f(z)}\right]_{z=e^{i \theta}}=\omega^{\prime}(\phi(\theta)) \cdot \phi^{\prime}(\theta),
$$

and thus $(\partial / \partial \theta)$ arg $\left(z f^{\prime}(z) / f(z)\right)_{z=r e^{\theta \theta}}$ has the continuous boundary function $\omega^{\prime}(\phi(\theta)) \phi^{\prime}(\theta)$ for $r=1$. Therefore we have

$$
\begin{aligned}
& \frac{d}{d \theta}\left[\log \left|\frac{z f^{\prime}(z)}{z}\right|\right]_{z=e^{i \theta}} \\
& \quad=\lim _{r \rightarrow 1} \frac{\partial}{\partial \theta}\left[\log \left|\frac{z f^{\prime}(z)}{f(z)}\right|\right]_{z=e^{i \theta}} \\
& =\frac{1}{2 \pi} \int_{0}^{\pi}\left[\omega^{\prime}(\phi(\theta+\tau)) \phi^{\prime}(\theta+\tau)-\omega^{\prime}(\phi(\theta-\tau)) \phi^{\prime}(\theta-\tau)\right] \cot \frac{\tau}{2} d \tau .
\end{aligned}
$$

This integral converges absolutely for every $\theta$ in the interval $0 \leqq \theta \leqq 2 \pi$. To see this we note that it may (formally) be written in the following way ( $\phi_{0}$ $=\phi(\theta))$ :

$$
\begin{aligned}
& -\frac{1}{2 \pi} \int_{0}^{\pi}\left[\omega^{\prime}(\phi(\theta+\tau))-\omega^{\prime}\left(\phi_{0}\right)\right] \phi^{\prime}(\theta+\tau) \cot \frac{\tau}{2} d \tau \\
& +\frac{1}{2 \pi} \int_{0}^{\pi}\left[\omega^{\prime}(\phi(\theta-\tau))-\omega^{\prime}\left(\phi_{0}\right)\right] \phi^{\prime}(\theta-\tau) \cot \frac{\tau}{2} d \tau \\
& -\frac{\omega^{\prime}\left(\phi_{0}\right)}{2 \pi} \int_{0}^{\pi}\left[\phi^{\prime}(\theta+\tau)-\phi^{\prime}(\theta-\tau)\right] \cot \frac{\tau}{2} d \tau .
\end{aligned}
$$

Hypothesis (b) and the fact that $\phi^{\prime}(\theta)$ is continuous imply that the first two

(16) This follows from Warschawski [11, Theorems III and IV] by noting the remarks on p. 320 under (3) Change of variables and on p. 318 under (1). 
integrals are absolutely convergent as is seen by introducing $x=\phi(\theta+\tau)$ and $x=\phi(\theta-\tau)$ respectively as variables of integration. The third integral converges absolutely since the existence of $\phi^{\prime \prime}(\theta)$ for every $\theta$ implies that $\lim _{\tau \rightarrow 0}\left(\left(\left(\phi^{\prime}(\theta+\tau)-\phi^{\prime}(\theta-\tau)\right) / \tau\right)\right)$ exists. If we make these substitutions, $x$ $=\phi(\theta+\tau)$ and $x=\phi(\theta-\tau)$, respectively, and note that the last integral is equal to $\omega^{\prime}\left(\phi_{0}\right) \cdot\left(\rho^{\prime} / \rho\right)\left[\phi_{0}\right] \cdot \phi^{\prime}(\theta)$, we are led to the formula

$$
\begin{aligned}
\frac{d}{d \theta}\left[\log \left|\frac{z f^{\prime}(z)}{f(z)}\right|\right]_{z=e^{i \theta}}= & -\frac{1}{2 \pi} \int_{0}^{2 \pi} \cot \frac{t(\phi)-\theta}{2}\left[\omega^{\prime}(\phi)-\omega^{\prime}\left(\phi_{0}\right)\right] d \phi \\
& +\omega^{\prime}\left(\phi_{0}\right) \cdot \frac{\rho^{\prime}}{\rho}\left[\phi_{0}\right] \cdot \phi^{\prime}(\theta) .
\end{aligned}
$$

Since (see, for example, (4.3))

$$
\log \phi^{\prime}(\theta)=\log \operatorname{Re}\left[\frac{z f^{\prime}(z)}{f(z)}\right]_{z=e^{i \theta}}=\left[\log \left|\frac{z f^{\prime}(z)}{f(z)}\right|\right]_{z=e^{i \theta}}+\log \cos \omega(\phi(\theta))
$$

we have

$$
\frac{\phi^{\prime \prime}(\theta)}{\phi^{\prime}(\theta)}=\frac{d}{d \theta}\left[\log \left|\frac{z f^{\prime}(z)}{f(z)}\right|\right]_{z=\theta^{i \theta}}-\tan \omega(\phi(\theta)) \cdot \omega^{\prime}(\phi(\theta)) \cdot \phi^{\prime}(\theta) .
$$

Hence

$$
\begin{aligned}
\frac{\phi^{\prime \prime}(\theta)}{\phi^{\prime}(\theta)}= & -\frac{1}{2 \pi} \int_{-\pi}^{\pi} \cot \frac{t(\phi)-\theta}{2}\left[\omega^{\prime}(\phi)-\omega^{\prime}\left(\phi_{0}\right)\right] d \phi \\
& +2 \omega^{\prime}\left(\phi_{0}\right) \cdot \frac{\rho^{\prime}}{\rho}\left[\phi_{0}\right] \cdot \phi^{\prime}(\theta) .
\end{aligned}
$$

(B) Preliminary inequalities. We first derive an upper bound for $\phi^{\prime}(\theta)$ using Theorem II. Hypothesis (a) implies

$$
\left|\frac{\rho^{\prime}}{\rho}[\phi]-\frac{\rho^{\prime}}{\rho}\left[\phi_{0}\right]\right| \leqq \frac{\epsilon}{\pi}\left|\phi-\phi_{0}\right| \leqq \epsilon
$$

if $\left|\phi-\phi_{0}\right| \leqq \pi$. Since $\int_{0}^{2 \pi}\left(\rho^{\prime} / \rho\right)[\phi] d \phi=0$, there exists a point $\phi_{1}$ such that $\left(\rho^{\prime} / \rho\right)\left[\phi_{1}\right]=0$. Hence, applying (5.4) with $\phi_{0}=\phi_{1}$ and using the periodicity of $\left(\rho^{\prime} / \rho\right)[\phi]$, we find for all $\phi, 0 \leqq \phi \leqq 2 \pi$,

$$
\left|\frac{\rho^{\prime}}{\rho}[\phi]\right|<\epsilon .
$$

Furthermore, by (5.4), we have $\left({ }^{17}\right)$

(17) Since the function $v(t)=t / \sin t$ when $t \neq 0, v(0)=0$ is concave (upward) for $0 \leqq t \leqq \pi / 2$, application of the trapezoidal rule for the approximate calculation of integrals shows that $\int^{\pi / 2}(t / \sin t) d t<2$. 


$$
\begin{aligned}
\frac{1}{2 \pi} \int_{\phi_{0}-\pi}^{\phi_{0}+\pi}\left|\frac{\left(\rho^{\prime} / \rho\right)[\phi]-\left(\rho^{\prime} / \rho\right)\left[\phi_{0}\right]}{\sin \left(\left(\phi-\phi_{0}\right) / 2\right)}\right| d \phi & \leqq \frac{1}{2 \pi} \cdot \frac{\epsilon}{\pi} \cdot 2 \int_{\pi_{0}}^{\phi_{0}+\pi}\left|\frac{\phi-\phi_{0}}{\sin \left(\left(\phi-\phi_{0}\right) / 2\right.}\right| d \phi \\
& =\frac{4 \epsilon}{\pi^{2}} \int_{0}^{\pi / 2} \frac{t}{\sin t} d t<\frac{8 \epsilon}{\pi^{2}} .
\end{aligned}
$$

Thus, the hypotheses of Theorem II are satisfied, $\delta$ being equal to $8 \epsilon / \pi^{2}$, and

$$
\left|\phi^{\prime}(\theta)-1\right| \leqq \frac{\left(8 / \pi^{2}\right) \epsilon+\epsilon^{2}}{1-\left(8 / \pi^{2}\right) \epsilon}
$$

or

$$
M^{*}=\max _{0 \leqq \theta \leqq 2 \pi} \phi^{\prime}(\theta) \leqq \frac{1+\epsilon^{2}}{1-\left(8 / \pi^{2}\right) \epsilon}<\frac{1+\epsilon^{2}}{1-(8 / 9) \epsilon} .
$$

(C) Estimate of $\left|\phi^{\prime \prime}(\theta)\right|$. Let $\phi_{1}$ be so chosen that $t\left(\phi_{1}\right)-\theta=\pi$ where $\phi_{1}$ $-2 \pi<\phi_{0} \leqq \phi_{1}$. Then $t\left(\phi_{1}-2 \pi\right)-\theta=-\pi$, and we may take the limits of integration in the integral of (5.3) as $\phi_{1}-2 \pi$ and $\phi_{1}$. For $\phi_{1}-2 \pi \leqq \phi \leqq \phi_{1},|t(\phi)-\theta|$ $\leqq \pi$ and, therefore,

$$
\left|\cot \frac{t(\phi)-\theta}{2}\right| \leqq \frac{2}{|t(\phi)-\theta|} \leqq \frac{M^{*}}{\left|\left(\left(\phi-\phi_{0}\right) / 2\right)\right|} \leqq \frac{M^{*}}{\left|\sin \left(\left(\phi-\phi_{0}\right) / 2\right)\right|}
$$

$\left(\phi \neq \phi_{0}\right)$

where $M^{*}$ is defined in (5.6). Hence by hypothesis (b)

$$
\begin{aligned}
\mid \frac{1}{2 \pi} \int_{\phi_{1}-2 \pi}^{\phi_{1}} \cot \frac{t(\phi)-\theta}{2}\left[\omega^{\prime}(\phi)\right. & \left.-\omega^{\prime}\left(\phi_{0}\right)\right] d \phi \mid \\
& \leqq \frac{M^{*}}{2 \pi} \int_{\phi_{1}-2 \pi}^{\phi_{1}}\left|\frac{\omega^{\prime}(\phi)-\omega^{\prime}\left(\phi_{0}\right)}{\sin \left(\left(\phi-\phi_{0}\right) / 2\right)}\right| d \phi \leqq M^{*} \epsilon,
\end{aligned}
$$

and thus from (5.3) by (5.7), hypothesis (a), and (5.5)

$$
\left|\frac{\phi^{\prime \prime}(\theta)}{\phi^{\prime}(\theta)}\right| \leqq M^{*}\left(\epsilon+\frac{2 \epsilon^{2}}{\pi}\right) \leqq\left(\frac{2}{1-\left(8 / \pi^{2}\right)}\right)\left(1+\frac{2}{\pi}\right) \epsilon .
$$

Making use of (5.6) we obtain (2.5).

(D) Estimate of $\left|f^{\prime \prime}(z) / f^{\prime}(z)\right|$. To prove (2.6) we note first that

$$
\begin{aligned}
\left|\frac{\partial}{\partial \theta} F(z)\right| & \equiv\left|i\left[1-z \frac{f^{\prime}(z)}{f(z)}\right]+i z \frac{f^{\prime \prime}(z)}{f^{\prime}(z)}\right| \\
& \leqq\left|\frac{\partial}{\partial \theta} \operatorname{Re} F(z)\right|+\left|\frac{\partial}{\partial \theta} \operatorname{Im} F(z)\right|
\end{aligned}
$$

for $z=r e^{i \theta}, 0 \leqq r \leqq 1$. If we estimate $\left|(d / d \theta)\left(\log \left|z f^{\prime}(z) / f(z)\right|_{z=e^{i \theta}}\right)\right|$ by use of 
(5.2), (5.7), hypothesis (a), (5.5), and (5.6), and if we estimate $\left|(d / d \theta)\left(\arg \left(z f^{\prime}(z) / f(z)\right)\right)_{z=e^{i \theta}}\right|$ by use of (5.1), (5.6), and hypothesis (a), we find

$$
\left|\frac{d}{d \theta} F\left(e^{i \theta}\right)\right| \leqq M^{*} \epsilon\left(1+\frac{\epsilon}{\pi}\right)+M^{*} \frac{\epsilon}{\pi}=h(\epsilon) .
$$

This inequality is true also when $|z|<1$, as may be seen in the following manner. Application of the mean value of the real and imaginary part of $F\left(e^{i \theta}\right)$ shows that for $r=1$

$$
\left|F\left(r e^{i(\theta+\gamma)}\right)-F\left(r e^{i \theta}\right)\right| \leqq h(\epsilon) \cdot|\gamma| \text {. }
$$

By the maximum modulus principle this is also true when $0 \leqq r<1$. Keeping $\theta$ and $r(0<r<1)$ fixed and letting $\gamma \rightarrow 0$, we find

$$
\lim _{\gamma \rightarrow 0}\left|\frac{F\left(r e^{i(\theta+\gamma)}-F\left(r e^{i \theta}\right)\right.}{\gamma}\right|=\left|\frac{\partial}{\partial \theta} F\left(r e^{i \theta}\right)\right| \leqq h(\epsilon) \quad(0 \leqq r \leqq 1) .
$$

By Theorem II (2.2) we have, using $\delta=\left(8 / \pi^{2}\right) \epsilon, 0<\epsilon<1$,

$$
\left|z \frac{f^{\prime}(z)}{f(z)}-1\right|<\frac{2 \epsilon+\epsilon^{2}+\epsilon^{3}}{1-\left(8 / \pi^{2}\right) \epsilon} \text {. }
$$

Hence, from (5.9) and (5.10), for $|z| \leqq 1$

$$
\begin{aligned}
\left|\frac{f^{\prime \prime}(z)}{f^{\prime}(z)}\right| & <\frac{2 \epsilon+\epsilon^{2}+\epsilon^{3}}{1-\left(8 / \pi^{2}\right) \epsilon}+h(\epsilon) \\
& \leqq \epsilon \cdot \frac{3+(1 / \pi)+\epsilon\left[\left(1+\epsilon+\epsilon^{2}\right) / \pi+1+2 \epsilon\right]}{1-\left(8 / \pi^{2}\right) \epsilon} \\
& <\epsilon \cdot \frac{3+(1 / \pi)+4 \epsilon}{1-\left(8 / \pi^{2}\right) \epsilon} .
\end{aligned}
$$

The proof of Theorem $\mathrm{V}$ is by induction and will be omitted. The ideas involved in the proof are essentially the ideas used in the proof of Theorem IV.

\section{BIBLIOGRAPHY}

1. Ludwig Bieberbach, Über die konforme Kreisabbildung nahezu kreisformiger Bereichen Sitzungsberichte der Preussichen Akademie der Wissenschaften, Mathematisch-Physikalishe Klasse (1924) pp. 181-188.

2. J. Ferrand, Sur la déformation analytique d'un domaine, C. R. Acad. Sci. Paris vol. 221 (1945) pp. 132-134.

3. F. Höhndorf, Verfahren zur Berechnung des Auftriebes gegebener Tragfächen-Profile, Zeitschrift für Angewandte Mathematik und Mechanik vol. 6 (1926) pp. 265-283.

4. A. R. Marchenko, Sur la représentation conforme, C. R. (Doklady) Acad. Sci. URSS. vol. 1 (1935) pp. 289-290. 
5. F. Riesz and M. Riesz, Über die Randwerte einer analytischen Funktion, Comptes Rendus du Quatrième Congrès des Mathématiciens Scandinaves à Stockholm, 1916, pp. 27-44. $87-95$.

6. F. Riesz, Über die Randwerte analytischer Funktionen, Math. Zeit. vol. 17 (1923) pp.

7. W. Seidel, Über die Ränderzuordnung bei konformen Abbildungen, Math. Ann. vol. 104 (1931) pp. 183-243.

8. T. Theodorsen, Theory of wing sections of arbitrary shape, National Advisory Committee for Aeronautics Technical Report no. 411, 1931.

9. T. Theodorsen and I. E. Garrick, General potential theory of arbitrary wing sections, National Advisory Committee for Aeronautics Technical Report no. 452, 1934.

10. S. E. Warschawski, Über das Randverhalten der Ableitung der Abbildungsfunktion bei konformer Abbildung, Math. Zeit. vol. 35 (1932) pp. 321-456.

11. - On the higher derivatives at the boundary in conformal mapping, Trans. Amer. Math. Soc. vol. 38 (1935) pp. 310-340.

12. - On Theodorsen's method of conformal mapping of nearlycircular regions, Quarterly of Applied Mathematics vol. 3 (1945) pp. 12-28.

Emmanuel Missionary College, Berrien Springs, Mich. 